Pacific Journal of Mathematic 


\section{PURITY AND ALGEBRAIC COMPACTNESS FOR MODULES}

R. B. WARFIELD, JR.

A submodule $A$ of a left module $B$ (over an associative ring with 1 ) is pure if for any right module $F$, the natural homomorphism $F \otimes A \rightarrow F \otimes B$ is injective. A module $C$ is pure-injective if for any module $B$ and pure submodule $A$, any homomorphism from $A$ to $C$ extends to $B$. The theory of this notion of purity and the corresponding class of pureinjectives is developed in this paper, with special attention to modules over commutative Noetherian rings and Prüfer rings. It is proved that pure-injective envelopes exist and the pureinjective modules are characterized as retracts of topologically compact modules. For this reason, the pure-injective modules are also called algebraically compact. For modules over Prüfer rings, certain simplifications occur, due essentially to the fact that a finitely presented module is a summand of a direct sum of cyclic modules. Complete sets of invariants are obtained for certain classes of algebraically compact modules over certain Prüfer rings.

This work is an extension of the theory of algebraically compact Abelian groups due to Kaplansky [8], Łoś [10], Maranda [12] and others. Our notion of algebraic compactness agrees with that introduced for general algebraic systems by Mycielski [14] and studied by Weglorz [20]. Related topics in module theory have been discussed by Fuchs [5], Fieldhouse [4], and Stenström [17], and there is some overlap between these papers and the results in the first and third sections below.

In the first section below, we discuss several notions of purity and identify the pure-projective modules in the cases which are of interest to us. In the second section we study finitely presented modules over a Prüfer ring, and use them to give a characterization of Prüfer rings. In the next two sections we consider the general theory of algebraically compact modules over arbitrary associative rings with 1. Sections five and six are devoted to the special results obtainable when the rings are commutative Noetherian rings and Prüfer rings respectively. All rings in this paper are associative with 1. We adopt the notation of [11] in using the arrow $\longmapsto$ for a monomorphism and $\longrightarrow$ for an epimorphism.

1. Purity and pure-projectives. Let $S$ be a class of left $R$ modules. We say a short exact sequence $A \nrightarrow B \rightarrow \mathrm{C}$ is $S$-pure if for 
any $M \in S$, the natural homomorphism $\operatorname{Hom}(M, B) \rightarrow \operatorname{Hom}(M, C)$ is surjective. We also say that in this situation $\mathrm{A}$ is an $S$-pure submodule of B. A module $P$ is $S$-pure-projective if for any $S$-pure short exact sequence $A \nrightarrow B \rightarrow C$, the natural homomorphism

$$
\operatorname{Hom}(P, B) \rightarrow \operatorname{Hom}(P, C)
$$

is surjective. Similarly, $D$ is $S$-pure-injective if for any such sequence, the natural homomorphism $\operatorname{Hom}(B, D) \rightarrow \operatorname{Hom}(A, D)$ is surjective. The $S$-pure projectives can be described in very general circumstances, the best results being contained in [15]. We prove the specific case that we will need.

Proposition 1. Let $S$ be a class of left $R$-modules, containing The module $R^{+}$( $R$ regarded as a left module over itself), such that there is a subclass $S^{*}$, which is a set, with the property that for any $M \in S$ there is an $N \in S^{*}$ with $N \cong M$. Then for any module $A$, there is an $S$-pure sequence $K \nrightarrow F \rightarrow A$, such that $F$ is a direct sum of copies of modules in $S$, and a module $P$ is $S$-pure-projective if and only if it is a summand of a direct sum of copies of modules in $S$.

REMARK. The set-theoretic hypothesis here can be weakened, but not simply removed, as examples in [15] show.

Proof. Let $\Lambda$ be the set of pairs $(M, f)$ with $M \in S^{*}$ and $f \in \operatorname{Hom}(M, A)$, and for each $\lambda \in \Lambda$, denote the corresponding $M$ and $f$ by $M_{\lambda}$ and $f_{\lambda}$. Let $F=\sum_{\lambda \in A} M_{\lambda}$ and let $\phi: F \rightarrow A$ be the homomorphism defined by the coordinate functions $f_{\lambda}$. Since $R^{+} \in S, \phi$ is surjective, and if $K$ is the kernel of $\phi$, it is clear by construction that $K$ is $S$-pure in $F$. For the second statement of the proposition, we apply the above result with $P=A$, and observe that if $P$ is $S$ pure-projective then $P$ is isomorphic to a summand of $F$.

The elements of $\operatorname{Ext}(A, B)$ corresponding to $S$-pure short exact sequences form a subgroup of $\operatorname{Ext}(A, B)$ which we denote by $\mathrm{P} \operatorname{ext}_{S}(A, B)$ and we note that

$$
\operatorname{Pext}_{S}(A, B)=\cap_{\lambda \in A} \operatorname{Ker}\left(f_{\lambda}^{*}\right)
$$

where $f_{2}^{*}$ is the natural homomorphism $\operatorname{Ext}(A, B) \rightarrow \operatorname{Ext}\left(M_{2}, B\right)$ induced by $f_{\lambda}$. It is easy to verify that the $S$-pure short exact sequences form a proper class in the sense of [11, 367-375], from which it follows that one can define the functors $\mathrm{P} \operatorname{ext}_{s}^{n}(A, B)$ for all $n>0$ in such a way that one gets the usual long exact sequences [11, 371375]. We omit the details in all of this since it will not play a significant role in what follows. We will be interested in two special cases of this general theory, and these cases are described in Pro- 
positions 2 and 3 below.

Proposition 2. The following properties of a short exact sequence $A \nrightarrow B \rightarrow C$ of $R$-modules are equivalent:

(a) For any $r \in R$, the natural homomorphism $\operatorname{Hom}(R / R r, B) \rightarrow$ $\operatorname{Hom}(R / R r, C)$ is surjective.

(b) For any $r \in R$, the natural homomorphism $R / r R \otimes A \rightarrow$ $R / r R \otimes B$ is injective.

(c) For any $r \in R, r A=A \cap r B$.

Proof. Note first that (a) holds if and only if for any $x \in B$ with $r x \in A$, there is a $y \in A$ with $r y=r x$. This makes the equivalence of (a) and (c) clear. To see the equivalence of (b) and (c) we simply chase the following commutative diagram with exact rows

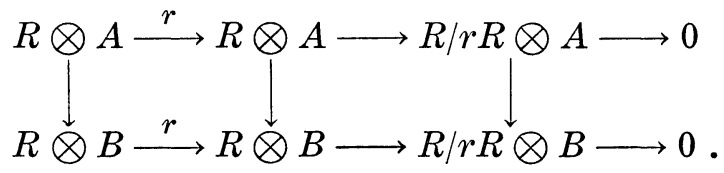

(Here, the map $r: R \otimes B \rightarrow R \otimes B$ denotes the map $r \otimes 1_{B}$ where $r: R \rightarrow R$ is left multiplication by $r$-a right module homomorphism.)

Definition. If a short exact sequence satisfies the conditions of the previous proposition then $A$ is said to be relatively divisible in $B$ and the short exact sequence is $R D$-pure. The corresponding projectives and injectives will be referred to as $R D$-projectives and $R D$ injectives.

This notion fits into the context of Proposition 1 if we (somewhat artificially) let $R D$ be the class of cyclic modules isomorphic to modules of the form $R / R r$. We can therefore derive the following corollary.

COROLlary 1. A left $R$-module is RD-projective if and only if it is a summand of a direct sum of cyclic modules of the form $R / R r$ (for various $r \in R$ ).

CoROllary 2. If $R$ is a commutative local ring then any $R D$ projective module is a direct sum of cyclic modules of the form $R / R r$ (for various $r \in R$ ).

Proof. If $P$ is $R D$-projective then there is a $Q$ such that $P \oplus Q=\sum_{i \in I} C_{i}$, where for each $i \in I$ there is an $r \in R$ with $C_{i}=$ $R / R r$. By [18, Proposition 3] it follows that $P=\sum_{j \in J} D_{j}$ where each 
$D_{j}$ is isomorphic to one of the $C_{i}$. (The key point is that $C_{i}$ has local endomorphism ring).

We recall that an $R$-module $M$ is finitely presented if there is a finitely generated free module $F$ and an epimorphism $F \rightarrow M$ whose kernel is finitely generated (that is, $M$ has a finite number of generators and a finite number of relations). We denote by $\operatorname{Hom}\left(R^{k}, R^{n}\right)$ the group of right-module homomorphisms of $R^{k}$ into $R^{n}$ and note that any element $\mu \in \operatorname{Hom}\left(R^{k}, R^{n}\right)$ can be regarded as an $n \times k$ matrix of elements of $R$. Any such $\mu$ induces a group homomorphism (which we also call $\mu$ ) $A^{k} \rightarrow A^{n}$ (for any left-module $A$ ) by identifying $A^{k}=$ $R^{k} \otimes A$, and taking the homomorphism $\mu \otimes 1_{\wedge}$.

Proposition 3. The following properties of a short exact sequence $A \nrightarrow B \rightarrow C$ of left $R$-modules are equivalent:

(a) For any finitely presented module $M$, the natural homomorphism $\operatorname{Hom}(M, B) \rightarrow \operatorname{Hom}(M, C)$ is surjective.

(b) For any right $R$-module $F$, the natural homomorphism $F \otimes A \rightarrow F \otimes B$ is injective.

(c) For any $\mu \in \operatorname{Hom}\left(R^{k}, R^{n}\right)$ (as above), $\mu\left(A^{k}\right)=A^{n} \cap \mu\left(B^{k}\right)$ (as subgroups of $B^{n}$ ).

$\left(\mathrm{c}^{\prime}\right)$ Any finite set of linear equations over $R$ with constants in $A$ which is soluble in $B$ is soluble in $A$.

REMARK. The equivalence of (a), (b) and ( $\left.c^{\prime}\right)$ was observed by Fieldhouse [4] and Stenström [17]. $\left(c^{\prime}\right)$ is just the specialization to modules of the general definition of purity used for algebraic systems by Weglorz [20].

Proof. If we look at the homomorphism $\mu$ as an $n \times k$ matrix, then the equivalence of $(c)$ and $\left(c^{\prime}\right)$ is a triviality.

Suppose our short exact sequence satisfies $\left(c^{\prime}\right)$ and $M$ is a finitely presented module and $f \in \operatorname{Hom}(M, C) . \quad M$ is defined by $n$ generators $x_{i}(1 \leqq i \leqq n)$ and $k$ relations $\sum_{i=1}^{n} r_{i j} x_{i}=0,(1 \leqq j \leqq k)$. Let $\phi: B \rightarrow$ $C$ be the epimorphism appearing in our short exact sequence, and choose elements $y_{i} \in B$ such that $\phi\left(y_{i}\right)=f\left(x_{i}\right), 1 \leqq i \leqq n$. The elements $y_{i}$ then satisfy the equations $\sum_{i=1}^{n} r_{i j} y_{i}=a_{j}(1 \leqq j \leqq k)$ for suitable $a_{j} \in A$. By $\left(\mathrm{c}^{\prime}\right)$ there are elements $z_{i} \in A$ satisfying the same equations. We now define $g: M \rightarrow B$ by $g\left(x_{i}\right)=y_{i}-z_{i}$. By construction, the $g$ so defined does yield a module homomorphism of $M$ into $B$ and it is clear that the image of $g$ under the natural homomorphism

$$
\operatorname{Hom}(M, B) \longrightarrow \operatorname{Hom}(M, C)
$$


is our original homomorphism $f$, so we have proved that $\left(\mathrm{c}^{\prime}\right)$ implies (a). Conversely, suppose that (a) is satisfied and that we have elements $y_{i} \in B(1 \leqq i \leqq n)$ satisfying the equations $\sum_{i=1}^{n} r_{i j} y_{i}=a_{j}$ for suitable $a_{j} \in A$, $(1 \leqq j \leqq k)$. We then define a finitely presented module $M$ with $n$ generators $x_{i}$ and $k$ relations $\sum_{i=1}^{n} r_{i j} x_{i}=0$, and applying the above argument backwards, we see that (a) implies $\left(c^{\prime}\right)$.

To prove the equivalence of (b) and (c) we first remark that it is enough to prove (b) for finitely presented modules $F$ since any module is a direct limit of finitely presented modules and tensor products commute with direct limits. Assuming, then, that $F$ is finitely presented, we take the first two steps of a free resolution of $F$ and tensor with $A$ and $B$ respectively obtaining the following commutative diagram with exact rows:

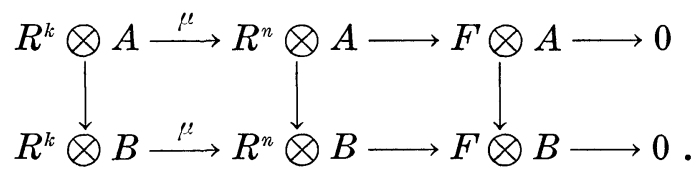

The equivalence of (b) and (c) is demonstrated by chasing the above diagram, where for any finitely presented $F$, the corresponding $\mu \in \operatorname{Hom}\left(R^{k}, R^{n}\right)$ is obtained from the free resolution of $F$ so that $F \cong$ Coker ( $\mu$ ) (where $F$ has $n$ generators and $k$ relations) and conversely, for any such $\mu$, we obtain a finitely presented $F$ by setting $F=\operatorname{Coker}(\mu)$.

DEFINITION. A short exact sequence satisfying the conditions of Proposition 3 will be called pure (without prefix, since this is the case of primary interest) and the corresponding projective and injectives will be referred to as pure-projectives and pure-injectives.

If we let $F P$ be the class of finitely presented left $R$-modules, then $F P$-purity in the sense of Proposition 1 coincides with purity as defined here and the conditions of Proposition 1 are satisfied.

CoRollary 3. A left $R$-module is pure-projective if and only if it is a summand of a direct sum of finitely presented modules.

Corollary 4. If $R$ is a complete, local, Noetherian, commutative ring then any pure-projective $R$-module is a direct sum of finitely generated modules.

Proof. Note first that finitely generated and finitely presented mean the same thing for modules over a Noetherian ring. By [3, Proposition 3.4] an indecomposable finitely generated module over such 
a ring has local endomorphism ring. Hence if $P$ is pure-projective, there is a $Q$ so that $P \oplus Q=\sum_{i \in I} C_{i}$ where each $C_{i}$ is a finitely generated module with local endomorphism ring. By [18, Theorem 1] it follows that there is a direct sum decomposition $P=\sum_{j \in J} D_{j}$ where each $D_{j}$ is isomorphic to one of the $C_{i}$.

2. Finitely presented modules over a Prüfer ring. We recall that a valuation ring is a (commutative) integral domain such that for any two elements $r$ and $s$, either $r$ divides $s$ or $s$ divides $r$. This clearly implies that any finitely generated ideal is principal (and hence projective) and that for any two ideals $I$ and $J$, either $I \subseteq J$ or $J \subseteq I$. In particular, a valuation ring is a local ring. A Prüfer ring is an integral domain such that every finitely generated ideal is projective. It is well known (for example by Corollary 2 above) that a local ring is a Prüfer ring if and only if it is a valuation ring, and, therefore, if $R$ is a Prüfer ring then for each maximal ideal $\mathfrak{m}, R_{\mathfrak{m}}$ is a valuation ring. It is also well known that this last fact characterizes Prüfer rings, but since a reference is not readily available, we include this in Proposition 5 below. We first need a local criterion for $R D$ projectivity.

Proposition 4. Let $R$ be a commutative ring and $M$ a finitely generated $R$-module. Then $M$ is $R D$-projective if and only if $M$ is finitely presented and for each maximal ideal $m$ of $R, M_{\mathfrak{m}}$ is a direct sum of cyclic $R_{\mathfrak{m}}$-modules of the form $R_{\mathfrak{m}} / R_{\mathfrak{m}} r\left(r \in R_{\mathfrak{m}}\right)$.

Proof. We first note that if $M$ is finitely generated and $R D$ projective, then $M$ is finitely presented since it is a summand of a finite direct sum of cyclic modules with principal annihilator ideals, and this direct sum is clearly finitely presented. If $M$ is $R D$-projective then $M_{\mathfrak{m}}$ is trivially $R D$-projective over $R_{\mathfrak{m}}$ and hence (by Corollary 2) a direct sum of cyclic modules of the form $R_{\mathfrak{m}} / R_{\mathfrak{m}} r$.

If $M$ is a finitely presented $R$-module and $N$ is arbitrary, then by [1, p. 98, Proposition 19] there is a natural isomorphism

$$
\operatorname{Hom}(M, N)_{\mathfrak{m}} \longrightarrow \operatorname{Hom}\left(M_{\mathfrak{m}}, N_{\mathfrak{m}}\right)
$$

where the module on the right represents the $R_{\mathfrak{m}}$-homomorphisms. We now assume that $M$ is finitely presented and $M_{\mathfrak{n}}$ is $R D$-projective for each maximal ideal $\mathrm{m}$. Let $A \nrightarrow B \rightarrow C$ be an $R D$-pure short exact sequence. Then for each $\mathfrak{m}$, the short exact sequence of $R_{\mathfrak{m}}$ modules $A_{\mathfrak{m}} \nrightarrow B_{\mathfrak{m}} \rightarrow C_{\mathfrak{m}}$ is $R D$-pure over $R_{\mathfrak{m}}$. (To see this note that any element $r^{*}$ of $R_{\mathfrak{m}}$ is of the form $r^{\prime} u$ where $u$ is a unit in $R_{\mathfrak{m}}$ and $r^{\prime}$ is the image of some element $r \in R$ under the natural map $R \rightarrow R_{\mathfrak{m}}$. 
Hence $R_{\mathfrak{m}} / r^{*} R_{\mathfrak{m}}=(R / r R)_{\mathfrak{m}}$ and the result follows by criterion (b) of Proposition 2.) Hence the natural homomorphism

$$
\operatorname{Hom}\left(M_{\mathfrak{m}}, B_{\mathfrak{m}}\right) \longrightarrow \operatorname{Hom}\left(M_{\mathfrak{m}}, C_{\mathfrak{m}}\right)
$$

is surjective for all $\mathrm{m}$. This together with the identification mentioned above shows that for each $\mathrm{m}$, the natural homomorphism

$$
\operatorname{Hom}(M, B)_{\mathfrak{m}} \longrightarrow \operatorname{Hom}(M, C)_{\mathfrak{m}}
$$

is surjective, and by [1, p. 111, Th. 1] this implies that the natural homomorphism

$$
\operatorname{Hom}(M, B) \longrightarrow \operatorname{Hom}(M, C)
$$

is surjective. Since this holds for all $R D$-pure short exact sequences, it follows that $M$ is $R D$-projective.

THEOREM 1. A finitely presented module over a valuation ring is a direct sum of cyclic modules. A finitely presented module over a Prüfer ring is a summand of a finite direct sum of cyclic modules. In each case, the cyclic modules can be chosen to be of the form $R / R r$ for some $r \in R$.

REMARK. The fundamental theorem of Abelian groups implies that every finitely generated module over a principal ideal domain is a direct sum of cyclic modules. Over a Dedekind domain the result is slightly weaker: any finitely generated module over a Dedekind domain is a summand of a finite direct sum of cyclic modules. This theorem is a natural generalization of this result (since a Noetherian ring is Prüfer if and only if it is Dedekind).

Proof. We first note that if $R / I$ is a cyclic module, then $R / I$ is finitely presented if and only if $I$ is finitely generated. This is not quite as obvious as it looks, but it follows from [1, p. 37, Lemma 9]. Now let $M$ be a finitely presented module over a valuation ring $R$ and let $z_{1}, \cdots, z_{n}$ be a set of generators for $M$. Suppose that of these $n$ elements, $z_{1}$ has the smallest order ideal. This implies that the cyclic submodule $S$ generated by $z_{1}$ is $R D$-pure. By induction on $n$ we may assume that $M / S$ is a direct sum of cyclic modules. Each of these cyclic modules must also be finitely presented and hence the order ideals must be finitely generated (by our first remark above) and therefore principal (since $R$ is a valuation ring). Hence $M / S$ is $R D$-projective, so that $M \cong S \oplus M / S$ which proves the first statement of the theorem. (This proof was partly modeled on the proof of [7, Th. 14].) 
Now let $M$ be a finitely presented module over a Prüfer ring $R$. For each maximal ideal $\mathfrak{m}, M_{\mathfrak{m}}$ is a finitely presented module over the valuation ring $R_{\mathfrak{m}}$, and hence a direct sum of cyclic $R_{\mathfrak{m}}$-modules. In particular, by the above argument, $M_{\mathfrak{m}}$ is $R D$-projective over $R_{\mathfrak{m}}$, so by Proposition $4, M$ is $R D$-projective over $R$, and hence a summand of a direct sum of cyclic modules. We can clearly specify that this sum be finite and that each of the cyclic summands be of the form $R / r R$ for some $r \in R$.

CoRollary 5. For modules over a Prüfer ring, purity and $R D$-purity coincide (by Theorem 1).

Proposition 5. For a commutative integral domain $R$, the following conditions are equivalent:

(a) For every maximal ideal $\mathfrak{m}, R_{\mathfrak{m}}$ is a valuation ring.

(b) Every finitely presented $R$-module is a summand of a direct sum of cyclic modules.

(c) Every finitely generated torsion-free module is projective.

(d) $R$ is a Prüfer ring.

Proof. That (c) implies (d) is trivial, and (d) implies (a) by our orignial remarks on Prüfer rings. The proof of Theorem 1 used only property (a) of Prüfer rings, and so shows that (a) implies (b). It remains to show that (b) implies (c), so suppose that (b) holds and $M$ is a finitely generated torsion-free module. It will suffice to show that $M$ is finitely presented. Let $x_{i}(1 \leqq i \leqq n)$ be a set of generators, and suppose that the $x_{i}(1 \leqq i \leqq k)$ are a maximal linearly independent subset of $M$. Then for each $i,(k<i \leqq n)$ there is a relation $r_{i} x_{i}=\sum_{j=1}^{k} r_{j i} x_{j}\left(r_{i} \neq 0\right)$, and we let $F$ be a module with $n$ generators satisfying just these relations. There is a natural epimorphism $F \rightarrow$ $M$. Since, by (b), $F$ is a summand of a direct sum of cyclic modules, the torsion submodule $T$ is a summand, and hence finitely generated, so $F / T$ is finitely presented. Since $F / T$ is torsion-free of rank $k$, the natural epimorphism $F / T \rightarrow M$ is an isomorphism, so $M$ is finitely presented, as desired.

3. Algebraic compactness. If $A$ is an Abelian group, there is a natural way to imbed $A$ into a compact Abelian group having certain universal properties. We let $T=R / Z$ (the circle group) and define the Bohr compactification $B(A)$

$$
B(A)=\operatorname{Hom}(\operatorname{Hom}(A, T), T) .
$$

Here we regard $\operatorname{Hom}(A, T)$ as a discrete Abelian group so that $B(A)$ 
has a natural compact Hausdorff topology. It is easy to show that $A$ is dense in $B(\mathrm{~A})$ and that any homomorphism of $A$ into a compact Abelian group $C$ extends to a unique continuous homomorphism of $B(A)$ into $C$. If we apply this remark to the homomorphism taking $A$ to $A / K$ (where $K$ is a subgroup) and thence to $B(A / K)$, we see that there is a natural isomorphism $B(A / K)=B(A) / \bar{K}$, where $\bar{K}$ denotes the closure of $K$ in $B(A)$. In particular, $\bar{K} \cap A=K$. Note also that if $A_{i}(i=1, \cdots, n)$ is a family of Abelian groups, then there is a natural isomorphism $B\left(\prod_{i=1}^{n} A_{i}\right)=\prod_{i=1}^{n} B\left(A_{i}\right)$.

Now suppose that $R$ is an associative ring with 1 and $A$ is a unitary left $R$-module. Then for any $r \in R$, the left multiplication by $r$ on $A$ extends uniquely to a continuous homomorphism of $B(A)$ into itself, making $B(A)$ a left $R$-module. It is not hard to see from what has gone before that $A$ is a pure submodule of the resulting $R$-module $B(A)$. Using criterion (c) of Proposition 3, we note that $\mu\left(B(A)^{k}\right)$ is just the closure of $\mu\left(A^{k}\right)$ in $B(A)^{n}=B\left(A^{n}\right)$, and using the fact that $\bar{K} \cap A^{n}=K$ for any subgroup of $A^{n}$ (where the closure is taken in $B\left(A^{n}\right)$ ) we have the desired result. We sum up the result in a definition and a lemma.

Definition. A left $R$-module $M$ is compact if there is a compact Hausdorff topology on $M$ making it a topological group and such that the left multiplications by elements of $R$ are continuous.

Note that we merely require the existence of such a topologyno further properties of it will be used.

Lemma 1. Any left $R$-module can be imbedded as a pure submodule in a compact module.

Definition. A left $R$-module $M$ is algebraically compact (Mycielski [14]) if every finitely soluble family of linear equations over $R$ in $M$ has a simultaneous solution.

The most useful interpretation of this condition is the following. Let $I$ be any index set (which will correspond to the set of variables) and consider the infinite product $M^{I}$. For any finite subset $I^{*} \subseteq I$, and elements $r_{i} \in R\left(i \in I^{*}\right)$ we can define a homomorphism $\phi: M^{I} \rightarrow M$ by $\phi(x)=\sum_{i \in I^{*}} r_{i} x_{i}$. (This is always a group homomorphism, and it is a module homomorphism if $R$ is commutative). By a linear equation we mean a pair $(\phi, m)$ where $\phi$ is a homomorphism of the type defined above and $m \in M$. The solution set of this equation $S(\dot{\phi}, m)$ is the set of all elements $x \in M^{I}$ such that $\phi(x)=m$. For a family of equations to be finitely soluble simply means that the corresponding sets 
$S(\phi, m)$ have the finite intersection property.

THEOREM 2. The following conditions on a left $R$-module $M$ are equivalent:

(a) $M$ is pure-injective.

(b) $M$ is a summand of a compact $R$-module.

(c) $M$ is algebraically compact.

Proof. If $M$ is pure-injective, then $M$ is a summand of a compact $R$-module, since by Lemma $1, M$ can be imbedded as a pure submodule in a compact module. Hence (a) implies (b). It is clear that a summand of an algebraically compact module is algebraically compact, so to show (b) implies (c) it suffices to show that a compact module is algebraically compact. If $M$ supports a compact Hausdorff topology then the product topology on $M^{I}$ is compact by the Tychonoff theorem, and since the sets $S(\dot{\phi}, m)$ of solutions are clearly closed, if they have the finite intersection property then their total intersection is nonempty, so a finitely soluble family of linear equations is soluble.

Finally, suppose that (c) holds and $A$ is an $R$-module, $B a$ pure submodule, and $f: B \rightarrow M$ a homomorphism. We take the elements of $A$ as our index set (or variables) and if $x \in M^{A}$, we denote the $a$ coordinate of $x$ by $x(a)$. We consider the equations

$$
r_{1} x\left(a_{1}\right)+r_{2} x\left(a_{2}\right)-x\left(r_{1} a_{1}+r_{2} a_{2}\right)=0
$$

(for any elements $a_{i} \in A, r_{i} \in R$ ) and $x(b)=f(b)$ for $b \in B$. The purity of $B$ in $A$ implies that these equations are finitely soluble (using criterion $\left(c^{\prime}\right)$ of Proposition 3), and hence this set of equations is soluble. Since a solution of this set of equations is simply a homomorphism from $A$ to $M$ extending $f$, we have shown that (c) implies (a).

Some historical notes are in order. This result was proved for Abelian groups by Łoś [10]. Algebraic compactness was defined for arbitrary algebraic systems by Mycielski [14] who showed that in general, (b) implies (c). The equivalence of (a) and (c) for general algebraic systems was shown by Weglorz [20], using the characterization $\left(\mathrm{c}^{\prime}\right)$ of purity. This was also shown independently for modules over Noetherian rings by Fuchs [5] and for modules over general rings by Stenstrom [17]. Each of these authors gives a proof for Corollary 6 and Proposition 6 below, Fuchs using reduced powers (and restricting to Noetherian rings) and Stenstrom using an adjoint functor theorem. The analogue of Corollary 6 does not hold for arbitrary algebraic systems, since W. F. Taylor has constructed noncommutative groups which cannot be imbedded in an algebraically compact group. 
CoRollary 6. Any left $R$-module can be imbedded as a pure submodule in a pure-injective module, (by Lemma 1 and Theorem 2).

Definitions. If $A$ is a pure submodule of $B$, then $B$ is a pureessential extension of $A$ if there are no nonzero submodules $S \subseteq B$, with $S \cap A=0$ and the image of $A$ pure in $B / S$. A pure extension $B$ of $A$ is a pure-injective envelope if $B$ is pure-injective and the extension is pure-essential.

Proposition 6. Pure-injective envelopes exist and are unique up to isomorphism. If $A$ is an $R$-module and $B$ a pure-injective envelope of $A$, and $f: A \rightarrow C$ an imbedding of $A$ as a pure submodule in a pure-injective module $C$, then $f$ extends to a homomorphism $B \rightarrow C$, imbedding $B$ as a pure submodule of $C$.

Proof. Let $A$ be a module and imbed $A$ as a pure submodule in a pure-injective module $C$. If $B$ is a pure-essential extension of $A$, then this imbedding extends to an imbedding of $B$ in $C$, from which it follows immediately that maximal pure-essential extensions exist. Let $B$ such an extension. Choose $S$, a submodule of $C$, maximal with respect to the properties that $S \cap A=0$ and the image of $A$ is pure in $C / S$. (It is necessary, but easy, to check that the union of an ascending family of submodules with these properties again has these properties.) If we regard $B$ as imbedded in $C$, then $B \cap S=0$ and since $C / S$ is (by construction) a pure-essential extension of the image of $A$, it follows that the image of $B$ is all of $C / S$, so that $C=S \oplus B$, and $B$ is pure-injective. Hence a maximal pure-essential extension is a pure-injective envelope. It follows immediately that if $C$ is any pure-injective module and $f: A \rightarrow C$ imbeds $A$ as a pure submodule, that $f$ extends to an imbedding of $B$ in $C$, and by the previous argument this imbedding is necessarily pure. The uniqueness of $B$ up to isomorphism follows.

Proposition 7. Let $A$ be an $R$-module and $B$ an algebraically compact $R$-module. Then $\operatorname{Hom}(A, B)$ and $\operatorname{Ext}^{n}(A, B)$ are algebraically compact as Abelian groups, and if $R$ is commutative they are algebraically compact as $R$-modules.

Proof. By Theorem 2 there is a compact $R$-module $C$ such that $B$ is a summand of $C$. In this case, $\operatorname{Hom}(A, B)$ and $\operatorname{Ext}^{n}(A, B)$ are summands of $\operatorname{Hom}(A, C)$ and $\operatorname{Ext}^{n}(A, C)$ respectively, so it suffices to show that $\operatorname{Hom}(A, C)$ and $\operatorname{Ext}^{n}(A, C)$ are compact $R$-modules. If we give $A$ the discrete topology and $C$ its compact topology, then 
Hom $(A, C)$ has a natural topology making it a compact Hausdorff Abelian group, and a topological $R$-module if $R$ is commutative. (The standard proof $[9$, p. 64, (18.1) (b)] for Abelian groups, where $C$ is the circle group, carries over almost verbatim.) We also note that if $f: A \rightarrow A^{\prime}$ is a homomorphism, then the induced homomorphism $\operatorname{Hom}\left(A^{\prime}, C\right) \rightarrow \operatorname{Hom}(A, C)$ is continuous, so the image and kernel are also compact. We now refer to the standard computation of $\operatorname{Ext}^{n}(A, C)$ from a projective resolution of $A,[11, \mathrm{p} .89]$. This gives us a family of modules $P_{i}$ and homomorphisms $d_{i}: \operatorname{Hom}\left(P_{i}, C\right) \rightarrow \operatorname{Hom}\left(P_{i+1}, C\right)$ which are induced from homomorphisms of the modules $P_{i}$ and are therefore continuous. It follows by our above remark that $\operatorname{ker}\left(d_{n}\right)$ and $i m\left(d_{n-1}\right)$ are closed subgroups of $\operatorname{Hom}\left(P_{n}, C\right)$, and hence their quotient, $\operatorname{ker}\left(d_{n}\right) / i m\left(d_{n-1}\right)=\operatorname{Ext}^{n}(A, C)$, also has a compact topology and is hence algebraically compact as an Abelian group, and as an $R$-module if $R$ is commutative.

4. Completeness and linear compactness. If $M$ is a left $R$ module, the $R$-topology on $M$ is defined by taking as neighborhoods of 0 the subgroups $r M(r \in R, r \neq 0)$. An Abelian group is known to be algebraically compact if and only if it is a direct sum of a divisible (injective) group and a group which is complete and Hausdorff in the $Z$-topology. We will show that strong completeness conditions hold for algebraically compact modules in general, including completeness in the $R$-topology. If $M$ is an $R$-module and $X$ is a subset of $R$, then by $X M$ we mean the subgroup of $M$ generated by the elements of the form $r m, m \in M, r \in X$. If $X$ and $Y$ are two subsets of $R$, then we denote by $(X M): Y$ the set of $m \in M$ such that $r m \in X M$ for all $r \in Y$. In particular, we have $(0 M): Y$, the set of elements of $M$ annihilated by $Y$, also denoted $M[Y]$. Also note that $(X M): R=X M$, since $R$ has a unit.

Proposition 8. Let $M$ be an algebraically compact $R$-module, $X_{i}$ and $Y_{i}(i \in I)$ subsets of $R$, where the sets $X_{i}$ are finite, and $z_{i}(i \in I)$ a family of elements of $M$. Let $N_{i}=\left(X_{i} M\right): Y_{i}$. Then if the sets $z_{i}+N_{i}$ have the finite intersection property, their total intersection in nonempty.

Proof. The condition $y \in z_{i}+N_{i}$ can be expressed by the equations $s\left(y-z_{i}\right)=\sum_{r \in X_{i}} r y_{r}\left(s \in Y_{i}\right)$, a set of equations indexed by the elements of $Y_{i}$ in the variables $y, y_{r}$. Note that it is essential that $X_{i}$ be finite. The finite intersection property guarantees that this system of equations (indexed by pairs $(i, s), s \in Y_{i}$ ) is finitely soluble, and algebraic compactness then gives us a global solution, which shows that the total intersection is not empty. 
CoROLlary 7. An algebraically compact $R$-module is complete in the R-topology.

Proof. This follows from Proposition 8 by considering the family of subgroups $N_{r}=r M=(r M): R(r \in R, r \neq 0)$.

If the ring $R$ is commutative then for any subset $X$ of $R$ we have $X M=I M$ where $I$ is the ideal generated by $X$, and $M[X]=$ $M[I]$ - the annihilator of the ideal generated by $X$. Proposition 8 can be rephrased in terms of projective limits. An important special case is the following. Suppose that $R$ is a commutative ring and $I_{j}(j \in J)$ is a directed decreasing family of finitely generated ideals (that is, for any $j$ and $k$ in $J$, there is an $n \in J$ with $I_{n} \subseteq I_{j} \cap I_{k}$ ). Then if $M$ is an algebraically compact $R$-module, the natural homomorphism

$$
M \longrightarrow \lim _{\longleftarrow} M / I_{j} M
$$

is surjective.

Suppose that $M$ is a topological $R$-module, by which we mean a module equipped with a Hausdorff topology making it a topological group such that the multiplications by elements of $R$ are continuous. $M$ is linearly compact in this topology (Lefschetz [9], Zelinsky [21]) if whenever we have a family of closed submodules $N_{i}(i \in I)$ and a family $x_{i}(i \in I)$ of elements of $M$, such that the sets $x_{i}+N_{i}$ have the finite intersection property, their total intersection is nonempty. When we do not care what the topology is, we simply say $M$ is linearly compact.

Proposition 9. A lineary compact module over a commutative ring is algebraically compact. If $R$ is a commutative ring which is either Noetherian or a valuation ring, then $R$ is algebraically compact as a module over itself if and only if it is linearly compact in the discrete topology.

REMARK. We refer to Zelinsky [21] for a discussion of rings satisfying this hypothesis.

Proof. By [9, pp. 78,19] a product of linearly compact modules is linearly compact in the product topology. Using the description of algebraic compactness given before Theorem 2, this implies that a linearly compact module $M$ is algebraically compact, using the linear compactness of products of the form $M^{I}$. Hence if $R$ is a linearly compact commutative ring, then it is algebraically compact as a module over itself. In the Noetherian case, the converse follows by Proposition 8 , proving that $R$ is actually linearly compact in the discrete 
topology (since the only closed submodules are the ideals, which are of the form $X R$ for some finite set $X$ ). If $R$ is a valuation ring, then for any two ideals, there is a principal ideal containing one and contained in the other, and hence Proposition 8 again implies linear compactness in the discrete topology.

In several special cases below, we will be able to give completeness conditions which are equivalent to algebraic compactness, but in general we will not be able to do this. Weglorz [20] has shown that a module is algebraically compact if and only if it is a retract of each of its ultrapowers, and this very general completeness condition is perhaps the best one can do in general. In the following two sections we will be able to get better results for modules over Noetherian rings and Prüfer rings.

5. Modules over Noetherian rings. If $A$ is an Abelian group with no elements of infinite height (that is, it is Hausdorff is the $Z$ topology) then the pure-injective envelope of $A$ is just its completion in this topology. For a similar result for modules over a commutative Noetherian ring, we are forced to restrict ourselves to finitely generated modules.

Definition. Let $\Omega$ denote the set of maximal ideals of the commutative Noetherian ring $R$, and let $M$ be an $R$-module. The $\Omega$-adic topology is defined on $M$ by taking as neighborhoods of 0 the submodules $I M$, where $I$ is an ideal which is a finite intersection of powers of the maximal ideals. (More formally, we consider functions $s$ of finite support from $\Omega$ to the nonnegative integers and take ideals of the form $I(s)=\cap \mathfrak{m}^{s(\mathfrak{m})},(\mathfrak{m} \in \Omega)$.)

We should note that the intersection of these ideals is just their product by [1, p. 71, Proposition 3].

Proposition 10. Let $R$ be a commutative ring and $M$ a topological $R$-module equipped with the $\Omega$-adic topology. For each $\mathfrak{m} \in \Omega$, denote by $M(\mathfrak{m})$ the module $M$ equipped with the $\mathfrak{m}$-adic topology, and let $u: M \rightarrow \Pi M(\mathfrak{m})(\mathfrak{m} \in \Omega)$ be the diagonal homomorphism. Then $u$ is continuous and induces a topological isomorphism

$$
\hat{M} \longrightarrow\left(\prod_{\mathfrak{n} \in \Omega} M(\mathfrak{m})\right)^{\hat{}}=\prod_{\mathfrak{m} \in \Omega} \hat{M}_{\mathfrak{m}}
$$

(where the completion on the left is taken in the $\Omega$-adic topology, that in the center with respect to the product of the $\mathrm{m}$-adic topologies, and $M_{\mathfrak{m}}$ denotes the localization of $M$ at $\mathfrak{m}$ equipped with the $\mathfrak{m}$-adic topology). (Compare [2, p. 54, Proposition 17].) 
Proof. We first remark that the completions of $M(\mathfrak{m})$ and $M_{\mathfrak{m}}$ is the $\mathrm{m}$-adic topology are naturally isomorphic by [2, p. 56, Proposition 18]. The remainder of the right-hand equality above is given by the fact that the completion of a product of uniform spaces is the product of their completions. An appropriate form of the Chinese remainder theorem [1, p. 73, Proposition 6] shows that $u$ maps $M$ continuously onto a dense submodule of $\Pi M(\mathfrak{m})$ and that the induced topology on $M$ as a submodule is the same as the $\Omega$-adic topology, from which the rest of the proposition follows.

If the ring $R$ is Noetherian, then Proposition 8 implies that an algebraically compact module is complete in the $\Omega$-adic topology. Hence the following corollary:

COROLlaRy 8. If $R$ is a commutative Noetherian ring and $M$ is an algebraically compact $R$-module which is Hausdorff in its $\Omega$ adic topology, then $M$ is complete and is a product of algebraically compact modules over the local rings $R_{\mathfrak{m}}(\mathfrak{m} \in \Omega)$.

We do not know whether, conversely, any complete, Hausdorff module over a Noetherian ring is necessarily algebraically compact, but in certain cases we can prove this.

Theorem 3. Let $R$ be a commutative Noetherian ring, and $E$, $F, G$ three finitely generated $R$-modules. Then

(a) If $E \rightarrow F \rightarrow G$ is an exact sequence of $R$-modules, then the induced sequence $\hat{E} \rightarrow \hat{F} \rightarrow \widehat{G}$ is exact (where the completions are with respect to the $Q$-adic topology).

(b) There is a natural isomorphism $\hat{R} \otimes E \rightarrow \hat{E}$.

(c) $\hat{E}$ is the pure-injective envelope of $E$. In particular, $E$ is pure in $\hat{E}$.

(d) $\hat{R}$ is faithfully flat as an $R$-module.

Proof. By [2, p. 68, Th. 3, (i)] the condition (a) above holds if we take completions in the m-adic topology, for any ideal $\mathrm{m}$. Since, by Proposition 10, the $\Omega$-adic completion is just the product of the m-adic completions, the result also holds for the $\Omega$-adic completions. The proof of (b) is then identical to the proof of (ii) in the same theorem, and the proof of condition (iii) of that theorem shows that $\hat{R}$ is a flat $R$-module. That $\hat{R}$ is actually faithfully flat follows from Proposition 10 above and $[1$, p. 44, Proposition I, (d)]. It remains to prove (c).

To show $E$ is purely imbedded in $\hat{E}$, we must first show that the $\Omega$-adic topology is Hausdorff on $E$. By [2, p. 65, Proposition 5] if 
$x \in E$ is in the closure of 0 then for any maximal ideal $\mathfrak{m}$, there is an $r \in \mathfrak{m}$ with $(1-r) x=0$. It follows $x$ goes to zero in the natural homomorphism $M \rightarrow M_{\mathfrak{m}}$. Since this is true for all maximal ideals $\mathfrak{m}$, it follows that $x=0$ by $[1, \mathrm{p} .112$, corollary 1]. Hence the homomorphism $E \rightarrow \hat{E}$ is injective, and by (b) above, this is just the natural homomorphism $E \rightarrow E \otimes \hat{R}$. Since this holds for any finitely generated module, it applies to the module $F \otimes E$, where $F$ is another finitely generated module. Hence, for any finitely generated module $F$, the induced homomorphism $F \otimes E \rightarrow F \otimes \widehat{E}$ is injective, which shows that $E$ is pure in $\hat{E}$ by criterion (b) of Proposition 3.

We must show, finally, that $\hat{E}$ is algebraically compact. We first remark that an Artinian module is linearly compact [17, Proposition 5] and hence algebraically compact by Proposition 9 above. By [9, pp. 78, 31-33] an inverse limit of linearly compact modules is linearly compact (if all of the relevant homomorphisms are continuous) so the inverse limit of Artinian modules is algebraically compact. It remains to show, then, that if $I$ is one of the ideals appearing in the definition of the $\Omega$-adic topology, that $E / I E$ is Artinian. By an iterative argument, it suffices to show that if $E / I E$ is Artinian then $E / \mathrm{m} I E$ is Artinian for any $\mathfrak{m} \in \Omega$, and for this it suffices to show that $I E / \mathfrak{m} I E$ is Artinian. Since $I E$ is finitely generated, $I E / \mathrm{m} I E$ is finite dimensional over $R / \mathrm{m}$, and hence trivially Artinian. This completes the proof.

\section{Modules over Prüfer rings.}

Theorem 4. If $R$ is a Prïfer ring, then an R-module $M$ is algebraically compact if and only if for any family of submodules $N_{i}(i \in I)$, where $N_{i}=\left(r_{i} M\right):\left(s_{i}\right)$, for some elements $r_{i}$ and $s_{i}$ of $R$, and any family $x_{i}(i \in I)$ of elements of $M$, if the sets $x_{i}+N_{i}$ have the finite intersection property, then their total intersection is nonempty.

Proof. That an algebraically compact module satisfies this condition is guaranteed by Proposition 8. Conversely, if $M$ is a module satisfying this completeness condition, we will show that $M$ is a maximal pure-essential extension of itself, which implies that it is algebraically compact by Proposition 6. Suppose, then, that $M$ is imbedded as a pure submodule of a module $A$, and $x \in A, x \notin M$. We will find an element $y \in M$ such that for any $r \in R$, if $r x \in M$ then $r x=r y$, and if $r$ divides $s x+m$ (for some $s \in R, m \in M$ ) then $r$ also divides $s y+m$. This we do as follows. Let $\Lambda$ be the set of triples $(r, s, m)$, with $r, s \in R, m \in M$, such that $r$ divides $s x+m$. We then look at the family of equations 


$$
\begin{aligned}
r y & =r x \quad(\text { for all } r \in R \text { such that } r x \in M) \\
r z_{\lambda} & =s y+m \quad(\lambda \in \Lambda) .
\end{aligned}
$$

These equations are finitely soluble in $C$ (using criterion $\left(c^{\prime}\right)$ of Proposition 3) since $C$ is pure in $A$ and these equations are globally soluble in $A$ (by setting $y=x$ ). To show these equations are globally soluble in $M$, note that if $r y_{0}=r x$, then $r y=r x$ if and only if $y \in y_{0}+M[r]$ (where $M[r]=(0 M):(r)$ and is therefore a submodule of our desired type), and similarly, if $r z=s y_{0}+m$, then there exists a $z^{\prime}$ with $r z^{\prime}=s y+m$ if and only if $y \in y_{0}+(r M):(s)$. Hence we have a family of subsets of $M$ of the type described in the theorem, and the finite solubility of the equations implies that these subsets have the finite intersection property. We can therefore choose an element $y$ in the intersection of all of these sets, and this element $y$ satisfies our requirements.

We now let $S$ be the submodule of $A$ generated by $x-y$ and our construction guarantees that $S \cap M=0 . \quad M$ therefore projects to a submodule $M^{\prime}$ of $A / S$ isomorphic to $M . \quad M^{\prime}$ is pure in $A / S$, since if $m \in M$ and $m$ is congruent to $r w$ modulo $S$, then for some $s \in R$, $r w=m-s y+s x$, which by construction shows there is a $z \in M$ with $r z=m-s y+s y$ so $m$ is divisible by $r$ in $M$. (This is enough to guarantee purity, by Corollary 5 , since $R$ is a Prüfer ring). The conclusion of all this is that $A$ is not a pure-essential extension of $M$, and since $A$ was an arbitrary pure extension, this shows that $M$ is a maximal pure-essential extension of itself, and is therefore algebraically compact.

Proposition 11. If $M$ is an algebraically compact module over a Prüfer ring, then $M=E \oplus N$, where $E$ is injective and $N$ is an algebraically compact module with no nonzero elements of infinite height (that is, no nonzero elements divisible by all nonzero elements of $R$ ).

Proof. Let $M$ be any $R$-module, and $M^{*}$ the submodule of elements of infinite height. Note that $M / M^{*}$ has no nonzero elements of infinite height. If $N$ is the pure-injective envelope of $M / M^{*}$, and if $N^{*}$ is the submodule of elements of infinite height in $N$, then $N^{*}=0$, since $\left(M / M^{*}\right) \cap N^{*}=0$ and the image of $M / M^{*}$ is still pure in $N / N^{*}$, which implies $N^{*}=0$ since $N$ is a pure-essential extension of $M / M^{*}$. The imbedding of $M^{*}$ into its injective envelope $E\left(M^{*}\right)$ can be extended to a homomorphism of $M$ into $E\left(M^{*}\right)$, and we therefore have an imbedding $M \rightarrow E\left(M^{*}\right) \oplus N$. The module on the right is algebraically compact and a pure-essential extension of $M$, and therefore is the pure-injective envelope. 
We offer the above two results as our generalization of the theorem that an Abelian group is algebraically compact if and only if it is the direct sum of a divisible (injective) group and a group with no elements of infinite height which is complete in its $Z$-topology. We would also like to have analogues of two other results. The first is that an algebraically compact group with no elements of infinite height is a direct product of complete modules over the $p$-adic integers (see also Corollary 8 above). The second is that there is a complete classification of the complete modules (with no elements of infinite height) over the $p$-adic integers [8]. We will find a reasonable class of Prüfer rings (including the Dedekind rings) for which the first result carries over, but for the second result, we restrict ourselves to certain cases (Theorems 5 and 6).

We follow $\mathrm{E}$. Matlis in calling a domain $R$ h-local if each nonzero prime ideal of $R$ is contained in a single maximal ideal of $R$, and each nonzero element of $R$ is contained in only a finite number of maximal ideals of $R$. Matlis shows [13, pp. 44-47] that a domain $R$ is $h$-local if and only if every torsion $R$-module $T$ is isomorphic to $\sum T_{\mathfrak{m}}$, where $\mathfrak{m}$ ranges over all maximal ideals of $R$. We note in passing two important consequences of this result for an $h$-local Prüfes ring $R$. The first is that a finitely presented torsion $R$-module is a direct sum of cyclic modules (by Theorem 1). The second is that the injective $R$-modules can be completely classified. Any such module is of the form $T \oplus F$, where $F$ is torsion-free and $T$ is torsion. $F$ is essentially just a vector space over the quotient field of $R$, and hence is completely described by its dimension. $T$ is a direct sum of injective modules over the valuation rings $R_{\mathfrak{m}}$, and these have a reasonable set of invariants, by the results in [19].

Proposition 12. If $R$ is an h-local Prïfer ring and $M$ an algebraically compact $R$-module with no nonzero elements of infinite height, then $M$ is a product of algebraically compact modules over the rings $R_{\mathrm{m}}$ (where $\mathrm{m}$ ranges over all maximal ideals of $R$ ).

Proof. By Corollary 7 and the fact that $M$ has no nonzero elements of infinite height, we know that there is a natural isomorphism

$$
M \longrightarrow \lim M / r M
$$

where $r$ ranges over all nonzero elements of $R$, ordered by divisibility. Since $R$ is $h$-local, $M / r M$ is a direct sum of torsion modules over the rings $R_{\mathfrak{m}}$ (for maximal ideals $\mathfrak{m}$ ) and $(M / r M)_{\mathfrak{m}}=0$ for all but a finite number of maximal ideals $m$ (since $r$ is contained in only a finite number of maximal ideals). It follows by standard results on inverse 
limits that

$$
M=\prod_{\mathfrak{m} \in \Omega} \lim _{(M / r M)_{\mathfrak{m}}}
$$

so that $M$ is a product of $R_{\mathfrak{n}}$ modules (which are necessarily algebraically compact).

We now turn to the study of algebraically compact modules over a valuation ring, and we restrict ourselves to modules with no elements of infinite height.

THEOREM 5. A torsion-free, algebraically compact module with no elements of intinite height over a valuation ring $R$ is the pureinjective envelope of a direct sum of ideals, and the number of ideals of each isomorphism type form a complete set of invariants.

Proof. Let $C$ be the module and $B$ a pure submodule which is a direct sum of ideals and which is maximal in the sense that it is not a summand of a larger pure submodule $B^{*}$ with $B^{*}=B \oplus J$, and $J$ isomorphic to an ideal of $R$. C contains a pure-injective envelope $E$ of $B$, which is a summand of $C$, so that $C=E \oplus D$. We must show $D=0$. Let $x \in D, x \neq 0$ and let $J$ be the set of all $y \in D$ such that there are nonzero elements $r, s$ of $R$ with $r x=s y$. Since $C$ has no elements of infinite height, $J$ is isomorphic to an ideal of $R . \quad J$ is pure in $D$, and therefore $B \oplus J$ is pure, contradicting the maximality of $B$.

To show that we have a complete set of invariants, we should show that the number of ideals of each isomorphism type appearing is independent of the choices involved. (We remark that two ideals $I, J$ are module-isomorphic if and only if there are nonzero elements $r, s$ of $R$ which $r I=s J$.) For this proof we refer to [19, Corollary 4.4].

Let $R$ and $S$ be valuation rings and $S$ an extension of $R$ and let the maximal ideal of $R$ be $\mathrm{m}$. Then $S$ is an immediate extension if there is a one-to-one correspondence between the ideals of $R$ and $S$ (that is, for every ideal $I$ of $R, I S \cap R=I$ and all ideals of $S$ arise in this way) and if the natural homomorphism $R / \mathfrak{m} \rightarrow S / \mathfrak{m} S$ is an isomorphism. $R$ is a maximal valuation ring if it has no proper immediate extension, or equivalently [16, pp. 36-51] if $R$ is algebraically compact as a module over itself (using Proposition 9 above). Any valuation ring $R$ has an immediate extension $S$ which is maximal, but $S$ is not in general unique as a ring [6].

Proposition 13. Let $R$ be a valuation ring, $S$ a maximal immediate extension of $R$, and $I$ any ideal of $R$. Then $(S / I S)^{+}$is the 
pure-injective envelope of $(R / I)^{+}$(where the imbedding is the natural one and the superscript indicates that all objects involved are being considered as $R$-modules).

Proof. Let $M=(S / I S)^{+}$. It is clear that $M$ is a pure extension of $(R / I)^{+}$since $S$ is an immediate extension of $R$. If $s$ and $r$ are in $R$, then if $r$ divides $s, r M:(s)=M$. Otherwise, $s$ divides $r$ and $r M:(s)=$ $\left(s^{-1} r\right) M$. By Theorem 4, therefore, we need only show that for any family of elements $x_{i}$ of $M$ and $r_{i}$ of $R$, if the sets $x_{i}+r_{i} M$ have the finite intersection property, then $\cap\left(x_{i}+r_{i} M\right)$ is nonempty. Choose coset representatives $y_{i}$ in $S$ for the $x_{i}$. If any of the $r_{i}$ are in $I$ the result is trivial so assume not. Then $y_{i}+r_{i} S$ is the inverse image in $S$ of $x_{i}+r_{i} M$, so the $y_{i}+r_{i} S$ also have the finite intersection property. Since $S$ is algebraically compact, $\cap\left(y_{i}+r_{i} S\right)$ is nonempty, and hence so is $\cap\left(x_{i}+r_{i} M\right)$.

Since $(S / I S)^{+}$is algebraically compact, it contains a pure-injective envelope, $E$, of $(R / I)^{+}$, and $(S / I S)^{+}=E \oplus V$. We must show $V=0$. If $x \in V, x \neq 0$, there is no element $y \in(S / I S)^{+}$such that $x$ is a multiple of $y$ and such that $y \notin \mathfrak{m}(S / I S)^{+}$. Since there are no elements $x(x \neq 0)$ in $(S / I S)^{+}$with this property, we conclude that $V=0$.

CoROllary 9. If $R$ is a valuation ring, then the $R$-module structure of a maximal immediate extension of $R$ is uniquely determined (taking $I=0$ in Proposition 13).

THeOREM 6. Let $R$ be a valuation ring, $S$ a maximal immediate extension of $R, M$ a finitely generated $R$-module and $E$ the pureinjective envelope of $M$. Then $E \cong M \otimes S, E$ is a finite direct sum of modules isomorphic to $(S / I S)^{+}$(for various ideals $I$ ), and any two such decompositions are equivalent.

Proof. We note first that $M \otimes S$ can be regarded as a finitely generated $S$-module. Since $S$ is a maximal valuation ring, by $[7, \mathrm{Th}$. 14] $M \otimes S$ is a finite direct sum of cyclic $S$-modules. Interpreting all of these as $R$-modules, we see that $M \otimes S$ is a direct sum of $R$ modules isomorphic to $(S / I S)^{+}$(for various ideals $I$ ), as desired. By Proposition 13 , these are algebraically compact $R$-modules, so $M \otimes S$ is algebraically compact. The endomorphism ring of $(S / I S)^{+}$is a local ring, which proves that any two of these decompositions are equivalent by the Azumaya theorem or by [18, Theorem 1].

We still must show that $M \otimes S$ is the pure-injective envelope of $M$. For purity, we use criterion (b) of Proposition 3, and for any module $F$ we look at the homomorphism 


\section{$F \otimes M \longrightarrow F \otimes M \otimes S$}

induced by the natural homomorphism $M \rightarrow M \otimes S$. The injectivity of this follows from the purity of $R$ in $S$, by applying criterion (b) again using the module $F \otimes M$ instead of $F$. To show that $M \otimes S$ is a pure-essential extension of $M$, note that the induced $R$-homomorphism $M / \mathfrak{m} M \rightarrow(M \otimes S) / \mathfrak{m}(M \otimes S)$ is an isomorphism, and the result follows as in the proof of Proposition 13.

\section{BIBLIOGRAPHY}

1. N. Bourbaki, Algèbre Commutative, (Ch. 1 and 2) Paris, Hermann, 1961.

2. - Algèbre Commutative, (Ch. 3 and 4) Paris, Hermann. 1961.

3. S.U. Chase, Torsion-free modules over $K(x, y)$, Pacific J. Math. 12 (1962), 437-447.

4. D. J. Fieldhouse, Purity and flat covers (to appear).

5. L. Fuchs, Algebraically compact modules over Noetherian rings (to appear in the Indian J. Math.)

6. I. Kaplansky, Maximal fields with valuations, Duke Math. J. 9 (1942), 303-321.

7. - Modules over Dedekind rings and valuation rings, Trans. Amer. Math. Soc. 72 (1952), 327-340.

8. - Infinite Abelian groups, Ann Arbor, 1954.

9. S. Lefschetz, Algebraic topology, Colloquium Publications vol. 27, New York, 1942.

10. J. Loś, Abelian groups that are direct summands of every Abelian group which contains them as pure subgroups, Fund. Math. 44 (1957), 84-90.

11. S. MacLane, Homology, Berlin, Springer, 1963.

12. J. M. Maranda, On pure subgroups of Abelian groups, Archiv der Math. 11 (1960), $1-13$.

13. E. Matlis, Cotorsion modules, Mem. Amer. Math. Soc. 49 (1964).

14. J. Mycielski, Some compactifications of general algebras, Coll. Math. 13 (1964), $1-9$.

15. F. Richman, C. Walker, E. A. Walker, Projective classes of Abelian groups (to appear in the Proceedings of the Colloquium on Abelian Groups held in Montpellier, June 1967).

16. O. Schilling, Valuation theory, Mathematical Surveys no. 4, New York, 1950.

17. B. Stenström, Pure submodules, Arkiv för Mat. 7 (1967), 159-171.

18. R. B. Warfield, Jr., A Krall-Schmidt theorem for infinite sums of modules (to appear).

19. - Decompositions of injective modules (to appear)

20. B. Weglorz, Equationally compact algebras, I. Fund. Math. 59 (1966), 289-298.

21. D. Zelinsky, Linearly compact modules and rings, Amer. J. Math. 75 (1953), 79-90.

Received March 28, 1968.

New Mexico State University 



\section{PACIFIC JOURNAL OF MATHEMATICS}

\section{EDITORS}

\author{
H. ROYDEN \\ Stanford University \\ Stanford, California \\ R. R Phelps \\ University of Washington \\ Seattle, Washington 98105
}

\author{
J. Dugundui \\ Department of Mathematics \\ University of Southern California \\ Los Angeles, California 90007
}

RICHARD ARENS

University of California

Los Angeles, California 90024

\section{ASSOCIATE EDITORS}
E. F. BeCKENBACH
B. H. NeUmanN
F. WolF
K. YosidA

\section{SUPPORTING INSTITUTIONS}

\author{
UNIVERSITY OF BRITISH COLUMBIA \\ CALIFORNIA INSTITUTE OF TECHNOLOGY \\ UNIVERSITY OF CALIFORNIA \\ MONTANA STATE UNIVERSITY \\ UNIVERSITY OF NEVADA \\ NEW MEXICO STATE UNIVERSITY \\ OREGON STATE UNIVERSITY \\ UNIVERSITY OF OREGON \\ OSAKA UNIVERSITY \\ UNIVERSITY OF SOUTHERN CALIFORNIA
}

\author{
STANFORD UNIVERSITY \\ UNIVERSITY OF TOKYO \\ UNIVERSITY OF UTAH \\ WASHINGTON STATE UNIVERSITY \\ UNIVERSITY OF WASHINGTON

$* * * * *$
AMERICAN MATHEMATICAL SOCIETY
CHEVRON RESEARCH CORPORATION
TRW SYSTEMS
NAVAL WEAPONS CENTER

The Supporting Institutions listed above contribute to the cost of publication of this Journal, but they are not owners or publishers and have no responsibility for its content or policies.

Mathematical papers intended for publication in the Pacific Journal of Mathematics should be in typed form or offset-reproduced, double spaced with large margins. Underline Greek letters in red, German in green, and script in blue. The first paragraph or two must be capable of being used separately as a synopsis of the entire paper. It should not contain references to the bibliography. Manuscripts, in duplicate if possible, may be sent to any one of the four editors. Please classify according to the scheme of Math. Rev. 36, 1539-1546. All other communications to the editors should be addressed to the managing editor, Richard Arens, University of California, Los Angeles, California, 90024.

50 reprints are provided free for each article; additional copies may be obtained at cost in multiples of 50 .

The Pacific Journal of Mathematics is published monthly. Effective with Volume 16 the price per volume (3 numbers) is $\$ 8.00$; single issues, $\$ 3.00$. Special price for current issues to individual faculty members of supporting institutions and to individual members of the American Mathematical Society: $\$ 4.00$ per volume; single issues $\$ 1.50$. Back numbers are available.

Subscriptions, orders for back numbers, and changes of address should be sent to Pacific Journal of Mathematics, 103 Highland Boulevard, Berkeley, California, 94708.

PUBLISHED BY PACIFIC JOURNAL OF MATHEMATICS, A NON-PROFIT CORPORATION

Printed at Kokusai Bunken Insatsusha (International Academic Printing Co., Ltd.), 7-17. Fujimi 2-chome, Chiyoda-ku, Tokyo, Japan. 


\section{Pacific Journal of Mathematics}

Vol. 28, No. 3

May, 1969

Jon F. Carlson, Automorphisms of groups of similitudes over $F_{3} \ldots \ldots \ldots$

W. Wistar (William) Comfort, Neil Hindman and Stelios A. Negrepontis,



Archie Gail Gibson, Triples of operator-valued functions related to the unit

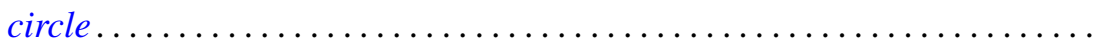

David Saul Gillman, Free curves in $E^{3}$

E. A. Heard and James Howard Wells, An interpolation problem for

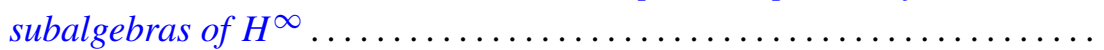

Albert Emerson Hurd, A uniqueness theorem for weak solutions of symmetric

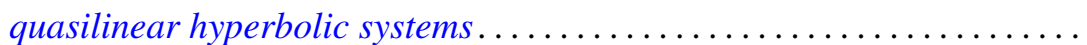

E. W. Johnson and J. P. Lediaev, Representable distributive Noether

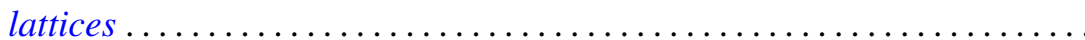

David G. Kendall, Incidence matrices, interval graphs and seriation in

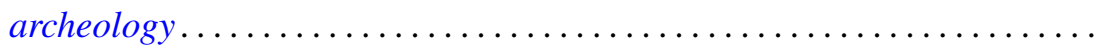
565

Robert Leroy Kruse, On the join of subnormal elements in a lattice ....... 571

D. B. Lahiri, Some restricted partition functions; Congruences modulo 3 .... 575

Norman D. Lane and Kamla Devi Singh, Strong cyclic, parabolic and conical differentiability........................................

William Franklin Lucas, Games with unique solutions that are

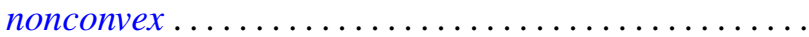

Eugene A. Maier, Representation of real numbers by generalized geometric series.

Daniel Paul Maki, A note on recursively defined orthogonal polynomials ...

Mark Mandelker, $F^{\prime}$-spaces and z-embedded subspaces ...

James R. McLaughlin and Justin Jesse Price, Comparison of Haar series with gaps with trigonometric series

Ernest A. Michael and A. H. Stone, Quotients of the space of irrationals ....

William H. Mills and Neal Zierler, On a conjecture of Golomb ...

J. N. Pandey, An extension of Haimo's form of Hankel convolutions ...

Terence John Reed, On the boundary correspondence of quasiconformal mappings of domains bounded by quasicircles...

Haskell Paul Rosenthal, A characterization of the linear sets satisfying Herz's criterion.

George Thomas Sallee, The maximal set of constant width in a lattice...

I. H. Sheth, On normaloid operators

James D. Stasheff, Torsion in BBSO ...

Billy Joe Thorne, A - P congruences on Baer semigroups.

Robert Breckenridge Warfield, Jr., Purity and algebraic compactness for

modules... 